PROCEEDINGS OF THE

AMERICAN MATHEMATICAL SOCIETY

Volume 131, Number 1, Pages 21-27

S 0002-9939(02)06619-4

Article electronically published on May 8, 2002

\title{
EQUIDISTRIBUTION OF HECKE EIGENFORMS ON THE MODULAR SURFACE
}

\author{
WENZHI LUO
}

(Communicated by Dennis A. Hejhal)

\begin{abstract}
For the orthonormal basis of Hecke eigenforms in $S_{2 k}(\Gamma(1))$, one can associate with it a probability measure $d \mu_{k}$ on the modular surface $X=$ $\Gamma(1) \backslash \mathbf{H}$. We establish that this new measure tends weakly to the invariant measure on $X$ as $k$ tends to infinity, and obtain a sharp estimate for the rate of convergence.
\end{abstract}

\section{INTRODUCTION}

Let $\left\{f_{j, k}\right\}_{1 \leq j \leq J_{k}}$ be the orthonormal basis of Hecke eigenforms in $S_{2 k}(\Gamma(1))$, the space of holomorphic cusp forms of weight $2 k$ with respect to the modular group $\Gamma(1)$. Thus

$$
J_{k}=\operatorname{dim}_{\mathbf{C}} S_{2 k}(\Gamma(1))= \begin{cases}{[k / 6]-1,} & \text { if } k \equiv 1(\bmod 6), \\ {[k / 6],} & \text { if } k \neq 1(\bmod 6) .\end{cases}
$$

One expects that the following equidistribution law holds for $f_{j, k}$ as the weight $k \rightarrow \infty$ : for any measurable subset $A$ on the modular surface $X=\Gamma(1) \backslash \mathbf{H}$, we have

$$
\left.\lim _{k \rightarrow \infty} \max _{1 \leq j \leq J_{k}}\left|\int_{A} y^{2 k}\right| f_{j, k}(z)\right|^{2} d \mu-\int_{A} d \mu \mid=0
$$

where

$$
d \mu=\frac{1}{\operatorname{area}(X)} \frac{d x d y}{y^{2}}
$$

is the normalized invariant measure on $X$.

The above statement can be viewed as a natural analogue of the unique quantum ergodicity conjecture, as formulated by Rudnick and Sarnak [2], for holomorphic cusp forms. While conjecture (1) seems out of reach at the present, one would like to establish this conjecture on the average. In [1] the variance over short intervals of $k$ has been studied.

Received by the editors August 6, 2001.

2000 Mathematics Subject Classification. Primary 11F11, 11F25.

Key words and phrases. Hecke eigenform, automorphic kernel.

This research was partially supported by NSF grant DMS-9988503, the Alfred P. Sloan Foundation Research Fellowship and the Seed Grant from the Ohio State University.

(C)2002 American Mathematical Society 
For each integer $k \geq 8$, which we are assuming throughout the paper, we associate with it a probability measure on $X$,

$$
d \mu_{k}=\frac{\sum_{j=1}^{\operatorname{dim} S_{2 k}(\Gamma(1))} y^{2 k}\left|f_{j, k}(z)\right|^{2}}{\operatorname{dim} S_{2 k}(\Gamma(1))} d \mu .
$$

The purpose of this simple note is to establish the following.

Theorem. For any measurable subset $A$ on the modular surface $X$ and any $\epsilon>0$, we have

$$
\int_{A} d \mu_{k}=\int_{A} d \mu+O_{\epsilon}\left(k^{-1 / 2+\epsilon}\right)
$$

uniformly for all $A$ on $X$.

Remark 1. What seems new here, at least for the non-compact modular surface, is the sharp decay rate $k^{-1 / 2+\epsilon}$. The stronger statement that

$$
\left.\sum_{j=1}^{J_{k}}\left|\int_{A} y^{2 k}\right| f_{j, k}(z)\right|^{2} d \mu-\left.\int_{A} d \mu\right|^{2} \ll k^{\epsilon}
$$

seems out of reach at the present.

Remark 2. One notes that $d \mu_{k}$ is actually independent of the choice of orthonormal basis.

Remark 3. Our proof is direct, without employing the theory of L-functions or imposing any conditions on $A$ (other than the measurability). The key ingredient is the holomorphic (automorphic) kernel $h_{k, m}\left(z, z^{\prime}\right)$ (see $\left.\S 2\right)$ for the Hecke operator $T_{k}(m)$.

2. HOLOMORPHIC KERNEL $h_{k, m}\left(z, z^{\prime}\right)$ FOR $T_{k}(m)$

The Hecke operator $T_{k}(m)(m \geq 1)$ acts on cusp form $f \in S_{2 k}(\Gamma(1))$ by

$$
T_{k}(m) f=\frac{1}{m} \sum_{a d=m} a^{2 k} \sum_{0 \leq b<d} f\left(\frac{a z+b}{d}\right) .
$$

It was shown by Zagier (see [3]) that $T_{k}(m)$ can be represented by the holomorphic automorphic kernel $C_{k}^{-1} m^{2 k-1} h_{k, m}\left(z, z^{\prime}\right)\left(C_{k}\right.$ defined in (7) below),

$$
h_{k, m}\left(z, z^{\prime}\right)=\sum_{a d-b c=m}\left(c z z^{\prime}+d z^{\prime}+a z+b\right)^{-2 k}
$$

where the sum is taken over all integer matrices $\left(\begin{array}{ll}a & b \\ c & d\end{array}\right)$ with determinant $m$, in the sense that

$$
\left\langle f, C_{k}^{-1} m^{2 k-1} h_{k, m}\left(\cdot,-\overline{z^{\prime}}\right\rangle_{k}=\left(T_{k}(m) f\right)\left(z^{\prime}\right)\right.
$$

for any $f \in S_{2 k}(\Gamma(1))$, where $\langle,\rangle_{k}$ is the (normalized) Petersson inner product on $S_{2 k}(\Gamma(1))$.

The series in (4) is absolutely convergent and $h_{k, m}\left(z, z^{\prime}\right)$ as a function of each variable $z$ or $z^{\prime}$ is a cusp form in $S_{2 k}(\Gamma(1))$, and we have the identity

$$
C_{k}^{-1} m^{2 k-1} h_{k, m}\left(z, z^{\prime}\right)=\sum_{j=1}^{J_{k}} \lambda_{j, k}(m) f_{j, k}(z) f_{j, k}\left(z^{\prime}\right)
$$


where $\lambda_{j, k}(m)$ is the Hecke eigenvalue of $f_{j, k}$ under $T_{k}(m)$ and

$$
C_{k}=\frac{3(-1)^{k}}{2^{(2 k-3)}(2 k-1)} .
$$

In particular, for $m=1$ and $z^{\prime}=z$, we obtain

$$
C_{k}^{-1} h_{k, 1}(z,-\bar{z})=\sum_{j=1}^{J_{k}}\left|f_{j, k}(z)\right|^{2} .
$$

The kernel $h_{k, m}\left(z, z^{\prime}\right)$ was used in 3 to give a new proof of Eichler-Selberg trace formula on $\Gamma(1)$. This note is motivated by 3 .

\section{Proof of the theorem}

Throughout we denote $\Gamma=\Gamma(1)$ and let $\chi_{A}$ denote the characteristic function of $A$ on $X$. One can extend it (with the same notation) to $\mathbf{H}$ as a $\Gamma$-invariant function. We have

$$
\begin{aligned}
\int_{A} d \mu_{k} & =\frac{1}{J_{k}} \int_{X} \chi_{A}(z)\left(\sum_{j=1}^{J_{k}} y^{2 k}\left|f_{j, k}(z)\right|^{2}\right) d \mu \\
& =\frac{1}{J_{k} C_{k}} \int_{X} \chi_{A}(z) h_{k, 1}(z,-\bar{z}) y^{2 k} d \mu \\
& =\frac{1}{J_{k} C_{k}} \int_{X} \chi_{A}(z)\left(\sum_{a d-b c=1} \frac{y^{2 k}}{\left(c|z|^{2}+d \bar{z}-a z-b\right)^{2 k}}\right) d \mu
\end{aligned}
$$

Since replacing $z$ by $\gamma z(\gamma \in \Gamma)$ in each term of the sum in (9) amounts to replacing

$$
\left(\begin{array}{ll}
a & b \\
c & d
\end{array}\right) \text { by } \gamma^{-1}\left(\begin{array}{ll}
a & b \\
c & d
\end{array}\right) \gamma
$$

we may decompose the sum into $\Gamma$-invariant pieces with $a+d=t, t \in \mathbf{Z}$. Thus,

$$
\int_{A} d \mu_{k}=\sum_{t=-\infty}^{\infty} \frac{1}{J_{k} C_{k}} \int_{X} \chi_{A}(z)\left(\sum_{a d-b c=1, a+d=t} \frac{y^{2 k}}{\left(c|z|^{2}+d \bar{z}-a z-b\right)^{2 k}}\right) d \mu .
$$

There is a bijection between the integral matrices $\left(\begin{array}{ll}a & b \\ c & d\end{array}\right)$ with determinant 1 and trace $t$, and the set of integral binary quadratic forms $g$ with discriminant $\operatorname{disc}(g)=t^{2}-4$. The bijection is given by

$$
\begin{gathered}
\left(\begin{array}{ll}
a & b \\
c & d
\end{array}\right) \mapsto g(u, v)=c u^{2}+(d-a) u v-b v^{2}, \\
g(u, v)=\alpha u^{2}+\beta u v+\gamma v^{2} \mapsto\left(\begin{array}{cc}
(t-\beta) / 2 & -\gamma \\
\alpha & (t+\beta) / 2
\end{array}\right) .
\end{gathered}
$$

For $g(u, v)=\alpha u^{2}+\beta u v+\gamma v^{2}$ and $z=x+i y$, set

$$
R_{g}(z, t)=\frac{y^{2 k}}{\left(\alpha\left(x^{2}+y^{2}\right)+\beta x+\gamma-i t y\right)^{2 k}} .
$$


Then for $\gamma \in \Gamma$ we have (here we identify the quadratic form $g$ with the symmetric $2 \times 2$ matrix representing $g$ )

$$
R_{\gamma^{T} g \gamma}(z, t)=R_{g}(\gamma z, t),
$$

and (10) can be written as

$$
\int_{A} d \mu_{k}=\sum_{t=-\infty}^{\infty} \frac{1}{J_{k} C_{k}} \int_{X} \chi_{A}(z)\left(\sum_{\operatorname{disc}(g)=t^{2}-4} R_{g}(z, t)\right) d \mu
$$

where the sum is taken over all forms of discriminant $t^{2}-4$.

For each discriminant $D=t^{2}-4$ and a quadratic form $g$ of discriminant $D$, we let $\Gamma_{g}$ denote the isotropy group of elements leaving $g$ fixed, and observe that

$$
\begin{aligned}
\sum_{\operatorname{disc}(g)=D} R_{g}(z, t) & =\sum_{\operatorname{disc}(g)=D, \bmod \Gamma} \sum_{\gamma \in \Gamma_{g} \backslash \Gamma} R_{\gamma^{T} g \gamma}(z, t) \\
& =\sum_{\operatorname{disc}(g)=D, \bmod \Gamma} \sum_{\gamma \in \Gamma_{g} \backslash \Gamma} R_{g}(\gamma z, t),
\end{aligned}
$$

where mod $\Gamma$ means the sum is taken over a set of representatives for classes of quadratic forms with discriminant $D$. For $D \neq 0$, recall the class number $h(D)$ is finite, and thus we obtain

$$
\int_{X} \chi_{A}(z)\left(\sum_{\operatorname{disc}(g)=D} R_{g}(z, t)\right) d \mu=\sum_{\operatorname{disc}(g)=D, \bmod \Gamma} \int_{X_{g}} \chi_{A}(z) R_{g}(z, t) d \mu
$$

where

$$
X_{g}=\bigcup_{\gamma \in \Gamma_{g} \backslash \Gamma} \gamma X
$$

is a fundamental domain for the action of $\Gamma_{g}$ on $\mathbf{H}$ with $X$ identified with a fundamental domain of $\Gamma$.

We distinguish the following three cases:

Case 1. $D=t^{2}-4<0$.

In this case $\left|\Gamma_{g}\right|=1,2$, or 3 and $t=0$, or \pm 1 . For a quadratic form

$$
g(u, v)=\alpha u^{2}+\beta u v+\gamma v^{2}
$$

with discriminant $D$, we have

$$
\begin{aligned}
\int_{X_{g}} \chi_{A}(z) R_{g}(z, t) d \mu & =\frac{1}{\left|\Gamma_{g}\right|} \int_{\mathbf{H}} \chi_{A}(z) R_{g}(z, t) d \mu \\
& =\frac{1}{\left|\Gamma_{g}\right|} \int_{\mathbf{H}} \chi_{A}((2 z-\beta) /(2|\alpha|)) \frac{y^{2 k}}{\left(|z|^{2} \pm i t y-D / 4\right)^{2 k}} d \mu
\end{aligned}
$$

where in the last step, we made the substitution $z \mapsto(2 z-\beta) /(2|\alpha|)$, and the sign ' \pm ' is ' - ' or ' + ' according to whether $\alpha>0$ or not.

Since

$$
\frac{2 y}{\left|\left(|z|^{2}-i t y-D / 4\right)\right|} \leq 1,
$$


we deduce that, with $D=t^{2}-4$ and $B_{k}=\left\{z=x+i y:|x| \leq k^{-1 / 2+\epsilon}, 0 \leq y \leq 3\right\}$,

$$
\begin{aligned}
& \sum_{D<0} \sum_{\operatorname{disc}(g)=D, \bmod \Gamma} \frac{1}{J_{k} C_{k}} \int_{X_{g}} \chi_{A}(z) R_{g}(z, t) d \mu \\
\ll & \int_{\mathbf{H}} \frac{(2 y)^{2 k}}{\left|\left(|z|^{2}-i t y-D / 4\right)\right|^{2 k}} d \mu \\
\ll & k^{-1 / 2+\epsilon}+\sup _{z \notin B_{k}} \frac{(2 y)^{2 k-2}}{\left|\left(|z|^{2}-i t y-D / 4\right)\right|^{2 k-2}} \times \int_{\mathbf{H}} \frac{(2 y)^{2}}{\left|\left(|z|^{2}-i t y-D / 4\right)\right|^{2}} d \mu \\
\ll & k^{-1 / 2+\epsilon}+\left(1-k^{-1+\epsilon}\right)^{k} \ll k^{-1 / 2+\epsilon} .
\end{aligned}
$$

In the last step above, the double integral converges and we estimate the function

$$
\frac{(2 y)^{2}}{\left|\left(|z|^{2}-i t y-D / 4\right)\right|^{2}}
$$

in the cases $\{z=x+i y: \quad y>3\}$ and $\left\{z=x+i y:|x|>k^{-1 / 2+\epsilon}, 0 \leq y \leq 3\right\}$ separately.

Case 2. $D=t^{2}-4=0$.

In this case, we can take as a system of representatives the forms $g_{r}(r \in \mathbf{Z})$, where $g_{r}(u, v)=r v^{2} . \Gamma_{g_{r}}$ is equal to $\Gamma$ for $r=0$, and is equal to

$$
\Gamma_{\infty}=\left\{ \pm\left(\begin{array}{cc}
1 & n \\
0 & 1
\end{array}\right)\right\}
$$

for $r \neq 0$. In the last case, in view of (16), we infer that

$$
\begin{aligned}
\int_{X} \chi_{A}(z)\left(\sum_{\operatorname{disc}(g)=0} R_{g}(z, t)\right) d \mu= & \int_{X} \chi_{A}(z) R_{g_{0}}(z, t) d \mu \\
& +\int_{X_{\infty}} \chi_{A}(z)\left(\sum_{r \neq 0} R_{g_{r}}(z, t)\right) d \mu,
\end{aligned}
$$

where $X_{\infty}$ is a fundamental domain for $\Gamma_{\infty}$, say the strip between 0 and 1.

First we have (recall $t= \pm 2$ )

$$
\frac{2}{J_{k} C_{k}} \int_{X} \chi_{A}(z) R_{g_{0}}(z, \pm 2) d \mu=\frac{2}{J_{k} C_{k}} \int_{A}\left(\frac{i}{2}\right)^{2 k} d \mu=\mu(A)+O\left(k^{-1}\right) .
$$

Next we deduce that

$$
\begin{aligned}
\frac{1}{J_{k} C_{k}} \int_{X_{\infty}} \chi_{A}(z)\left(\sum_{r \neq 0} R_{g_{r}}(z, t)\right) d \mu \\
=\frac{1}{J_{k} C_{k}} \int_{0}^{\infty} \int_{0}^{1} \chi_{A}(z) y^{2 k-2} \sum_{r \neq 0}(r-i t y)^{-2 k} d x d y \\
=\frac{1}{J_{k} C_{k}} \int_{0}^{k^{1 / 2-\epsilon}} \int_{0}^{1} \chi_{A}(z) y^{2 k-2} \sum_{r \neq 0}(r-i t y)^{-2 k} d x d y \\
\quad+\frac{1}{J_{k} C_{k}} \int_{k^{1 / 2-\epsilon}}^{\infty} \int_{0}^{1} \chi_{A}(z) y^{2 k-2} \sum_{r \neq 0}(r-i t y)^{-2 k} d x d y
\end{aligned}
$$


We infer that

$$
\begin{aligned}
& \frac{1}{J_{k} C_{k}} \int_{0}^{k^{1 / 2-\epsilon}} \int_{0}^{1} \chi_{A}(z) y^{2 k-2} \sum_{r \neq 0}(r-i t y)^{-2 k} d x d y \\
\ll & \max _{0 \leq y \leq k^{1 / 2-\epsilon}}\left(\frac{(2 y)^{2}}{1+(2 y)^{2}}\right)^{k-1} \int_{0}^{k^{1 / 2-\epsilon}}\left(\sum_{r \neq 0}\left(r^{2}+(2 y)^{2}\right)^{-1}\right) d y \ll k^{-1} .
\end{aligned}
$$

On the other hand, since

$$
\frac{\pi^{2}}{\sin ^{2}(\pi z)}=\sum_{r \in \mathbf{Z}}(r-z)^{-2}
$$

and for $s \neq 0$,

$$
\frac{d^{2 k-2}}{d s^{2 k-2}} \sinh ^{-2}(\pi s y)=4 \sum_{n \geq 1} n(2 \pi n y)^{2 k-2} \exp (-2 \pi n|s| y) \geq 0,
$$

we have

$$
\begin{aligned}
& \frac{1}{J_{k} C_{k}} \int_{k^{1 / 2-\epsilon}}^{\infty} \int_{0}^{1} \chi_{A}(z) y^{2 k-2}\left(\sum_{r \neq 0}(r-i t y)^{-2 k}\right) d x d y \\
= & \left.\frac{1}{J_{k} C_{k}} \frac{i^{2 k-2}}{(2 k-1) !} \int_{k^{1 / 2-\epsilon}}^{\infty} \int_{0}^{1} \chi_{A}(z) \frac{d^{2 k-2}}{d s^{2 k-2}}\right|_{s=t}\left(\sum_{r \neq 0}(r-i s y)^{-2}\right) d x d y \\
= & \left.\frac{1}{J_{k} C_{k}} \frac{i^{2 k-2}}{(2 k-1) !} \int_{k^{1 / 2-\epsilon}}^{\infty} \int_{0}^{1} \chi_{A}(z) \frac{d^{2 k-2}}{d s^{2 k-2}}\right|_{s=t}\left(\frac{1}{s^{2} y^{2}}-\frac{\pi^{2}}{\sinh ^{2}(\pi s y)}\right) d x d y \\
\ll & k^{-1 / 2+\epsilon}+\frac{1}{(2 k-1) !} \int_{k^{1 / 2-\epsilon}}^{\infty}\left(\sum_{n \geq 1} n(4 \pi n y)^{2 k-2} \exp (-4 \pi n y)\right) d y \\
\ll \quad & k^{-1 / 2+\epsilon}+k^{-1} \sum_{n \geq 1} \Gamma\left(2 k-1,4 \pi n k^{1 / 2-\epsilon}\right),
\end{aligned}
$$

where

$$
\Gamma(m, x)=\frac{1}{\Gamma(m)} \int_{x}^{\infty} e^{-\xi} \xi^{m-1} d \xi=e^{-x}\left(\frac{x^{m-1}}{(m-1) !}+\frac{x^{m-2}}{(m-2) !}+\cdots+x+1\right)
$$

is the incomplete $\Gamma$-function.

We deduce that

$$
\begin{aligned}
\sum_{n \geq 1} \Gamma\left(2 k-1,4 \pi n k^{1 / 2-\epsilon}\right)= & \sum_{n \leq k^{1 / 2+2 \epsilon}} \Gamma\left(2 k-1,4 \pi n k^{1 / 2-\epsilon}\right) \\
& +\sum_{n \geq k^{1 / 2+2 \epsilon}} \Gamma\left(2 k-1,4 \pi n k^{1 / 2-\epsilon}\right) \\
& \ll k^{1 / 2+2 \epsilon},
\end{aligned}
$$

in view of the estimates $0<\Gamma(m, x) \leq 1$ for $x \geq 0$, and

$$
\Gamma(m, x) \leq e^{-x} \frac{x^{m-1}}{(m-1) !}\left(1-\frac{m-1}{x}\right)^{-1}
$$

provided $x>m-1$. 
Thus

$$
\sum_{D=0} \frac{1}{J_{k} C_{k}} \int_{X} \chi_{A}(z)\left(\sum_{\operatorname{disc}(g)=0} R_{g}(z, t)\right) d \mu=\mu(A)+k^{-1 / 2+2 \epsilon} .
$$

Case 3. $D=t^{2}-4>0$.

Here again the class number is finite as in the case $D<0$, but the isotropy groups are infinite cyclic. Let $g(u, v)=\alpha u^{2}+\beta u v+\gamma v^{2}$ be a quadratic form with the discriminant $D$. The conjugate of $\Gamma_{g}$ by some $\gamma \in S L_{2}(\mathbf{R})$ acts on $\mathbf{H}$ as the infinite cyclic group generated by $z \mapsto \epsilon^{2} z$, where $\epsilon>1$ is the fundamental unit of the order in $\mathbf{Q}(\sqrt{D})$ associated with $g$. We have

$$
\left(\gamma^{T} g \gamma\right)(u, v)=\sqrt{D} u v
$$

and we can choose the fundamental domain $X_{g}$ such that $\gamma^{-1} X_{g}$ is an annulus defined by

We infer that

$$
\Delta: \quad y>0 ; \quad 0<r_{0} \leq|z| \leq \epsilon^{2} r_{0}
$$

$$
\begin{aligned}
\frac{1}{J_{k} C_{k}} \int_{X_{g}} \chi_{A}(z) R_{g}(z, t) d \mu & =\frac{1}{J_{k} C_{k}} \int_{X_{g}} \chi_{A}(z) R_{\gamma^{T} g \gamma}\left(\gamma^{-1} z, t\right) d \mu \\
& =\frac{1}{J_{k} C_{k}} \iint_{\Delta} \chi_{A}(\gamma z)(\sqrt{D} x-i t y)^{-2 k} y^{2 k-2} d x d y \\
& \ll \int_{0}^{\pi} \int_{r_{0}}^{\epsilon^{2} r_{0}}\left(\frac{4}{t^{2}-4 \cos ^{2} \theta}\right)^{k} \frac{d r}{r} d \theta \\
& \ll(\log |t|)(4 / 5)^{k} \frac{1}{\left(t^{2}-4\right)^{2}} .
\end{aligned}
$$

Since $h(D)=h\left(t^{2}-4\right) \ll|t|^{1+\epsilon}$, we conclude that

$$
\sum_{D>0} \sum_{\operatorname{disc}(g)=D, \bmod \Gamma} \frac{1}{J_{k} C_{k}} \int_{X_{g}} \chi_{A}(z) R_{g}(z, t) d \mu \ll(4 / 5)^{k} .
$$

This completes the proof of the theorem.

\section{ACKNowledgement}

The author would like to thank Peter Sarnak and Zeév Rudnick for some helpful comments.

\section{REFERENCES}

[1] W.Luo and P.Sarnak, Mass equidistribution for Hecke eigenforms, preprint, 2001.

[2] Z.Rudnick and P.Sarnak, The behaviour of eigenstates of arithmetic hyperbolic manifolds, Comm. Math. Phys. 161, 1994, 195-213. MR 95m:11052

[3] D.Zagier, The Eichler-Selberg trace formula on $S L_{2}(\mathbf{Z})$, Appendix to Introduction to Modular Forms by S.Lang, Springer, Berlin, 1976, 44-54; errata in Lecture Notes in Math., Vol. 627, Springer-Verlag, 171-173, 1977. MR 58:522

Department of Mathematics, The Ohio State University, 231 West 18th Avenue, Columbus, Ohio 43210

E-mail address: wluo@math.ohio-state.edu 